\title{
Efeito de três diferentes diluidores sobre o sêmen canino submetido a dois protocolos de descongelamento
}

Isabel Candia Nunes da CUNHA $^{1}$

Maria Denise LOPES²

\section{Correspondência para:}

ISABEL CANDIANUNES DACUNHA

Centro de Ciências e Tecnologias Agropecuárias

Laboratório de Melhoramento Genético Animal

Universidade Estadual do Norte

Fluminense "Darcy Ribeiro"

Avenida Alberto Lamego, 2000 -

Parque Califórnia

28013-600 - Campos dos Goytacazes-RJ

cunhaicn@uenf.br

Recebido para publicação: 27/11/2003 Aprovado para publicação: 15/03/2005

\author{
1 - Centro de Ciências e Tecnologias Agropecuárias do Laboratório de \\ Melhoramento Genético Animal da Universidade Estadual do Norte \\ Fluminense "Darcy Ribeiro" - UENF, Campos dos Goytacazes - RJ \\ 2 - Departamento de Reprodução Animal e Radiologia Veterinária \\ da Universidade Estadual Paulista - UNESP, Campus de Botucatu, Botucatu - SP
}

\section{Resumo}

Com objetivo de avaliar o efeito de dois protocolos de descongelação do sêmen canino congelado em três diluidores foram coletados ejaculados de nove cães. Cada ejaculado foi dividido em 3 amostras, centrifugado e os pellets tratados sob diferentes protocolos: $1^{\circ}$ - meio á base de glicina e gema de ovo; $2^{\circ}$ - meio á base de TRIS e $3^{\circ}$ - meio M9. Foram envasadas 28 palhetas de $0,5 \mathrm{ml}$ segundo cada protocolo. Amostras tratadas sob cada protocolo foram avaliadas (M1) quanto a motilidade, vigor e integridade das membranas. As palhetas foram refrigeradas a $5^{\circ} \mathrm{C}$ por $60 \mathrm{~min}$. e congeladas em N2. Uma amostra de cada protocolo foi avaliada quanto: motilidade, vigor e integridade das membranas após o equilíbrio (M2). Eram sempre descongeladas três pares de palhetas, sendo que cada par havia sido congelado sob um mesmo protocolo. Uma das palhetas de cada par era descongelada a $37^{\circ} \mathrm{C}$ por 30 segundos e outra a $72^{\circ} \mathrm{C}$ por 8 segundos. Cada grupo foi avaliado após o descongelamento (M3) quanto a motilidade e vigor espermático, avaliação computadorizada do movimento, integridade das membranas e avaliação acrossomal por meio de FitcPNA e PI. As amostras foram mantidas em banho Maria a $37^{\circ} \mathrm{C}$ por $1 \mathrm{~h}$ para o teste de longevidade espermática e reavaliadas (M4) quanto à motilidade e vigor espermáticos, integridade das membranas. Após análise estatística concluímos que as células espermáticas caninas descongeladas a $72^{\circ} \mathrm{C}$ por 8 segundos apresentaram um maior somatório de bons resultados quanto aos testes de viabilidade espermática in vitro empregados.

\section{Introdução}

A criopreservação e suas conseqüências sobre a célula espermática são os assuntos mais descritos e estudados pelos pesquisadores da área de biotecnologia do sêmen. Sabemos que tanto a refrigeração como a congelação e descongelação são eventos que podem culminar em alterações irreversíveis da célula espermática. São necessários ainda muitos estudos para que possamos compreender a origem destas alterações para que, num futuro, estes efeitos deletérios possam ser minimizados ou eliminados ${ }^{1,2}$.

Watson ${ }^{2}$ afirmou que as mudanças pós-ejaculatórias do espermatozóide no trato feminino em sua preparação para a fusão com o oócito devem ser vistas como a continuidade no desenvolvimento e maturação espermática, processo este que teve inicio no epidídimo. A criopreservação implica em uma pausa neste processo natural pelo qual o espermatozóide é "inativado", porém, ao invés de ser "reativado" no estágio de onde tinha parado, sua maturação é afetada pelo processo. Concluiu ainda que o processo de criopreservação faz com que os espermatozóides se tornem mais reativos pelo fato de suas membranas sofrerem mudanças na fluidez e tornarem-se mais 
permeáveis ao cálcio. Estes fenômenos podem promover a capacitação e reação do acrossomo, diminuindo o tempo de vida útil do espermatozóide, impossibilitando a futura fertilização do no local e momento apropriado.

O meio diluidor deve proteger as células espermáticas dos efeitos adversos de todo o processo de criopreservação. As propriedades protetoras de um meio diluidor são atribuídas aos diferentes componentes deste meio e da sua interação com as células espermáticas de uma determinada espécie, e ainda, de um determinado indivíduo ${ }^{2,3,4}$.

O processo de descongelamento parece ser tão deletério aos espermatozóides quanto a congelação e os principais efeitos adversos são principalmente atribuídos ao fenômeno de recristalização dos microcristais de gelo intracelulares podendo provocar ruptura da membrana plasmática devido a suas formas pontiagudas e a alterações bruscas no volume celular decorrentes da acomodação dos diferentes gradientes osmóticos gerados durante a congelação $0^{2,3,5,6}$.

Um protocolo de congelação e descongelação de sêmen deve incluir a temperatura e o período de exposição ideal, considerando-se, para tanto, as características particulares daquele protocolo. Várias temperaturas e tempos de exposição têm sido descritos para a descongelação do sêmen canino, assim como inúmeros protocolos de congelação e descongelação ${ }^{1,4,7,8,9}$. A descongelação com exposição a altas temperaturas por um curto período de tempo tem sido preconizada para a descongelação do sêmen canino ${ }^{1,4,7,9,10,11}$ conferindo longa viabilidade às amostras de sêmen tanto in vitro como in vivo. Em contrapartida, os protocolos mais utilizados para outras espécies animais, incluindo aquele preconizado pelo Ministério da Agricultura $^{12}$, indicam a descongelação de palhetas de $0,5 \mathrm{ml}$ à $37^{\circ} \mathrm{C}$ durante 30 segundos.

Após o processo de congelação e descongelação das células espermáticas, testes de alta correlação com a fertilidade devem ser realizados, para que se verifique a viabilidade dos diluidores e \ou dos métodos de congelação utilizados ${ }^{1,13}$.

O movimento retilíneo progressivo é considerado normal em cães, e reflete uma completa habilidade e viabilidade para fertilizar o ovócito ${ }^{4}$, por tanto, a avaliação subjetiva do movimento espermático é muito importante e deve ser rotineiramente realizada, porém, pelo fato de ser realizada por um técnico, pode estar sujeita a um grande número de variações, principalmente, no que diz respeito ao grau de habilidade $\mathrm{e}$ experiência do avaliador ${ }^{14}$. Para que estas variações sejam minimizadas uma avaliação computadorizada do movimento espermático pode ser realizada. Recentemente, Iguer-Ouada e Verstegen ${ }^{14}$ propuseram a padronização de um avaliador computadorizado de movimento espermático (HTR-IVOS10 analyzer, Hamilton Thorn Research, Beverly, Mass, USA) que apresentou ótimos resultados na avaliação de espermatozóides da espécie canina.

Métodos identificadores da integridade das membranas espermáticas têm sido utilizados na avaliação de diferentes técnicas de conservação do sêmen ${ }^{1,6,13,15,16,17}$. Cunha ${ }^{18}$ padronizou um teste para a verificação da integridade das membranas espermáticas de cães por meio de sondas fluorescentes, sendo elas o diacetato de carboxifluoresceína e o iodeto de propídeo.

$O$ isotiocianato fluorescinado (Fitc) é uma sonda fluorescente comum e largamente utilizada para verificação do estado acrossomal. O Fitc deve estar ligado a uma lecitina, que é uma proteína ou uma glicoproteina isolada de sementes de várias plantas. Estas lecitinas se ligam especificamente a resíduos de açúcares encontrados em locais específicos da célula. As duas lecitinas mais utilizadas na verificação de estado acrossomal são o PSA - aglutinina Pisum sativum oriunda da ervilha, que se liga à matriz acrossomal e o PNA - aglutinina Arachis hypogea originária do amendoim que se liga à membrana acrossomal externa $a^{1,5,13,19,20,21}$.

A alteração da fluidez das membranas 
espermáticas durante a capacitação propicia a fusão da membrana acrossomal externa com a membrana plasmática, este fenômeno fusogênico é conhecido como reação do acrossomo $^{13,19}$. Cross e Watson ${ }^{20}$ e Medeiros et al. ${ }^{21}$ utilizaram o Fitc-PNA para verificar o estado acrossomal de espermatozóides bovinos e eqüinos, respectivamente, e sugeriram que tal sonda fluorescente se liga a membrana acrossomal externa quando esta exibe uma alteração de permeabilidade. Para que se pudessem diferenciar os espermatozóides com a total perda do acrossomo dos acrossomos intactos utilizase o iodeto de propídio em conjunto com o Fitc PNA ${ }^{13,21}$.

Com vista no exposto tivemos como objetivo neste experimento avaliar o efeito de dois diferentes protocolos de descongelação do sêmen canino congelado em três diferentes diluidores sobre a qualidade do movimento espermático, integridade das membranas espermáticas e a condição acrossomal.

\section{Materiais e Métodos}

\section{Animais}

Foram coletados e processados ejaculados de nove cães (um ejaculado por cão) provenientes de criatórios particulares da cidade de Botucatu-SP. A colheita foi realizada por meio de massagem peniana.

\section{Exame do Sêmen}

Motilidade e vigor espermático: através de microscopia de contraste de fase e o resultado expresso em porcentagem (0-100) para a motilidade e através de um escore de zero a cinco $(0-5)^{12}$ para o vigor.

Integridade das membranas: pela associação do Iodeto de Propídio e

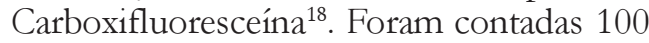
células e classificadas como: íntegras - células emitindo fluorescência verde ou lesadas células com o núcleo emitindo fluorescência vermelha.

Avaliação do estado acrossomal: pela associação do iodeto de propídio (PI) e do
Fitc-PNA. Foi realizada a contagem de 200 células espermáticas em campo claro e, imediatamente após, as mesmas células foram examinadas sob epifluorescência e classificadas como: reação acrossomal verdadeira - emitindo apenas fluorescência verde (Fitc-PNA); falsa reação do acrossomo - emitindo fluorescência verde na região acrossomal (Fitc-PNA) e vermelha (PI); espermatozóide lesados - emitindo apenas fluorescência vermelha (PI); espermatozóide intactos - células verificadas somente sob campo claro (não emitindo fluorescência).

\section{Procedimentos experimentais}

Depois de coletado e avaliado, cada ejaculado foi dividido em 3 amostras idênticas, acrescida, na proporção de 1:1, de meio à base de leite desnatado e glicose ${ }^{22}$ ( $\mathrm{pH}-7,26 ; 355 \mathrm{mOSM}$ ) e centrifugada por 5 min. a 800g. Os 3 "pellets" formados foram tratados sob diferentes protocolos segundo a metodologia descrita por Cunha e Lopes ${ }^{23}$ : GG - ressuspendido em meio á base de glicina e gema de ovo (GG) sem glicerol (pH 7; 285 mOSM) e após 5 min. acrescido de GG com 12,8\% de glicerol e envasado; TRIS - ressuspendido em meio á base de TRIS, acido cítrico e glicose (TRIS) com 3\% de glicerol ( $\mathrm{pH}$ 6,10; 788 mOSM) e após 5 min. acrescido de TRIS com 7\% glicerol e envasado e M9 - ressuspendido em meio M9 (33\% Merck-gema, 33\% Kenney, 33\% DME® (Sigma), 5\% glicerol na solução final) e envasado em palhetas de $0,5 \mathrm{ml}$, ficando este protocolo com 5\% de glicerol em sua concentração final. Foram envasadas 28 palhetas de $0,5 \mathrm{ml}$ segundo cada protocolo, ficando as palhetas com concentração espermática média de 33,73 milhões de espermatozóide/ palheta.

Amostras tratadas sob cada protocolo foram avaliadas (M1) quanto a motilidade e vigor espermáticos e integridade das membranas e levadas a um refrigerador computadorizado programado para a temperatura de $5^{\circ} \mathrm{C}$. Tal temperatura foi atingida após $20 \mathrm{~min}$. de refrigeração e permaneceu por mais $40 \mathrm{~min}$. nesta 
Tabela 1 - Medianas da motilidade espermática (\%) do sêmen canino congelado e descongelado avaliadas após a diluição (M1), após o período de equilíbrio (M2), após 10 min. da descongelação (M3) e após o teste de longevidade (M4), nos seis grupos propostos. Botucatu, 2002

\begin{tabular}{|c|c|c|c|c|c|c|}
\cline { 2 - 7 } \multicolumn{1}{c|}{} & G1 & G2 & G3 & G4 & G5 & G6 \\
\hline M1 & 70 & 70 & 80 & 80 & 80 & 80 \\
\hline M2 & 70 & 70 & 60 & 60 & 70 & 70 \\
\hline M3 & 40 & 50 & 40 & 50 & 50 & 40 \\
\hline M4 & 20 & 10 & 10 & 30 & 10 & 10 \\
\hline
\end{tabular}

temperatura para o equilíbrio ${ }^{23}$. Decorrido período de equilíbrio as palhetas foram levadas a uma caixa de isopor e mantidas a $4 \mathrm{~cm}$ do $\mathrm{N}^{2}$ por 20 min. e após mergulhadas no $\mathrm{N}^{2}$ e armazenadas em botijões apropriados $^{23}$. Uma amostra de cada protocolo foi levada diretamente do equilíbrio ao banho Maria $37^{\circ} \mathrm{C}$ por $10 \mathrm{~min}$. e avaliada (M2) quanto à motilidade e vigor espermáticos e integridade das membranas espermáticas, pelos métodos descritos anteriormente.

Na primeira etapa foram descongeladas 18 palhetas congeladas sob cada protocolo. Eram sempre descongeladas três pares de palhetas de uma mesma partida de congelação, sendo que cada par havia sido congelado sob um mesmo protocolo. Uma das palhetas de cada par foi descongelada a $37^{\circ} \mathrm{C}$ por 30 segundos e outra palheta descongelada a $72^{\circ} \mathrm{C}$ por 8 segundos formando os grupos: G1 - GG descongelado a $37^{\circ} \mathrm{C}$ por $30 \mathrm{seg}$; G2 - GG descongelado a $72^{\circ} \mathrm{C}$ por 8 seg; G3 - M9 descongelado a $37^{\circ} \mathrm{C}$ por $30 \mathrm{seg}$; G4 - M9 e descongelado a $72^{\circ} \mathrm{C}$ por 8 seg; G5 - TRIS descongelado a $37^{\circ} \mathrm{C}$ por $30 \mathrm{seg}$; G6 - TRIS e descongelado a $72^{\circ} \mathrm{C}$ por 8 segs.

Cada grupo foi avaliado quanto após o descongelamento (M3) à motilidade e vigor espermático e integridade das membranas. As amostras foram mantidas em banho Maria $37^{\circ} \mathrm{C}$ e, decorridos 30 min., em média, do descongelamento, foi realizada a avaliação da qualidade do movimento espermático através de avaliação computadorizada (Hamilton Thorn Research - IVOS 10) sendo verificados os
Tabela 2 - Medianas do vigor espermático (escore de 0 - 5) do sêmen canino congelado e descongelado avaliadas após a diluição (M1), após o período de equilíbrio (M2), após 10 min. da descongelação (M3) e após o teste de longevidade (M4), nos seis grupos propostos. Botucatu, 2002

\begin{tabular}{|c|c|c|c|c|c|c|}
\cline { 2 - 7 } \multicolumn{1}{c|}{} & G1 & G2 & G3 & G4 & G5 & G6 \\
\hline$M 1$ & $3 \mathrm{~A}$ & $3 \mathrm{~A}$ & $3 \mathrm{~A}$ & $3 \mathrm{~A}$ & $2 \mathrm{~B}$ & $2 \mathrm{~B}$ \\
\hline $\mathrm{M} 2$ & $3 \mathrm{~B}$ & $3 \mathrm{~B}$ & $3 \mathrm{~A}$ & $3 \mathrm{~A}$ & $2 \mathrm{C}$ & $2 \mathrm{C}$ \\
\hline $\mathrm{M} 3$ & 1 & $2^{*}$ & 2 & $2^{\otimes}$ & 1 & $2^{*}$ \\
\hline $\mathrm{M} 4$ & $1 \mathrm{~A}$ & $1 \mathrm{~A}$ & $0 \mathrm{~A}$ & $1 \mathrm{~A}$ & $0 \mathrm{AB}$ & $1 \mathrm{AB}$ \\
\hline
\end{tabular}

Letras maiúsculas diferentes em uma mesma linha indicam diferença estatística entre diluidores para cada temperatura de descongelação (G1 x G3 × G5) e (G2 x G4 × G6) (p<0,05).

$\ddot{\AA}$ indicatendência a superioridade estatística entre diluidores para cada temperatura de descongelação (G1 x G3 x G5) e (G2 × G4 × G6) $(0,05<p<0,10)$

*indica tendência a superioridade estatística entre temperaturas para cada diluidor (G1xG2), (G3xG4) e (G5xG6) $(0,05<p<0,10)$

parâmetros: motilidade total (MT), motilidade progressiva (MP), padrão médio de velocidade(VAP) e linearidade(LIN).

As amostras foram mantidas em banho Maria a $37^{\circ} \mathrm{C}$ por $1 \mathrm{~h}$ após o descongelamento para o teste de longevidade espermática e, após este período, reavaliadas (M4) quanto à motilidade e vigor espermáticos, integridade das membranas.

Posteriormente foram descongeladas 10 palhetas congeladas sob cada protocolo $(n=30)$ sendo cinco amostras para cada grupo descrito (G1, G2, G3 e G4) e nestas amostras realizada a avaliação do estado acrossomal.

\section{Métodos estatísticos utilizados}

Para a comparação entre dois momentos no mesmo tratamento: prova não paramétrica de Wilcoxon para pequenas amostras pareadas, com o cálculo da estatística p. Para a comparação entre 3 momentos no mesmo tratamento: prova não paramétrica de Friedman para amostras dependentes, com o cálculo das estatíticas c ${ }^{2}$ e p.

Para a comparação entre tratamentos em cada momento: prova não paramétrica de Friedmam com o cálculo das estatísticas $c^{2}$ e $p$.

As estatísticas calculadas foram consideradas significantes quando $\mathrm{p}<0,05$. 
Tabela 3 - Medianas da integridade das membranas espermáticas (\%) do sêmen canino congelado e descongelado avaliadas após a diluição (M1), após o período de equilíbrio (M2) após 10 min. da descongelação (M3) e após o teste de longevidade (M4), nos seis grupos propostos. Botucatu, 2002

\begin{tabular}{|c|c|c|c|c|c|c|}
\cline { 2 - 7 } \multicolumn{1}{c|}{} & G1 & G2 & G3 & G4 & G5 & G6 \\
\hline M1 & 86 & 86 & 87 & 87 & 91 & 91 \\
\hline M2 & 82 & 82 & 86 & 86 & 81 & 81 \\
\hline M3 & 53 & 57 & 53 & 62 & 59 & 62 \\
\hline M4 & $34 \mathrm{~B}$ & $37 \mathrm{~B}$ & $28 \mathrm{Ba}$ & $39 \mathrm{ABb}$ & $46 \mathrm{~A}$ & $44 \mathrm{~A}$ \\
\hline
\end{tabular}

Letras maiúsculas diferentes em uma mesma linha indicam diferença estatística entre diluidores para cada temperatura (G1 x G3 x G5) e (G2 x G4 x G6) $(p<0,05)$

Letras minúsculas diferentes em uma mesma linha indicam diferença estatística entre temperaturas para cada diluidor (G1xG2), (G3xG4) e (G5xG6) $(p<0,05)$

Nos casos em que $0,05<p<0,10$ foi referida tendência à significância, já que p é a probabilidade de erroneamente concluir pela significância.

\section{Resultados e Discussão}

O resfriamento, congelação e descongelação preservam a célula espermática, porém inúmeros danos são verificados após a descongelação ${ }^{2,3}$. Comprovamos que houve uma diminuição de viabilidade espermática após a congelação e descongelação, com diminuição de motilidade e vigor espermático e da integridade das membranas espermáticas em

Tabela 4 - Mediana da qualidade do movimento espermático descrito por motilidade total (MT), motilidade progressiva (MP), velocidade média (VAP) e linearidade do movimento (LIN) avaliada após 30 min. da descongelação, nos seis grupos propostos. Botucatu, 2002

\begin{tabular}{|c|c|c|c|c|c|c|}
\cline { 2 - 7 } \multicolumn{1}{c|}{} & $\mathrm{G} 1$ & $\mathrm{G} 2$ & $\mathrm{G} 3$ & $\mathrm{G} 4$ & $\mathrm{G} 5$ & $\mathrm{G} 6$ \\
\hline MT & $20 \mathrm{~A}$ & $24 \mathrm{~A}$ & $16 \mathrm{~B}$ & $17 \mathrm{~B}$ & $11 \mathrm{~B}$ & $23 \mathrm{~B}$ \\
\hline MP & $10 \mathrm{~A}$ & $22 \mathrm{~A}^{*}$ & $10 \mathrm{~B}$ & $9 \mathrm{~B}$ & $3 \mathrm{~B}$ & $11 \mathrm{~B}^{*}$ \\
\hline VAP & 83,4 & $89,6 \mathrm{AB}^{*}$ & 72 & $78,4 \mathrm{~B}$ & 73,7 & $79,2 \mathrm{~B}^{*}$ \\
\hline LIN & $62 \mathrm{~A}$ & $71 \mathrm{~A}^{*}$ & $68 \mathrm{~A}$ & $64 \mathrm{~B}$ & $57 \mathrm{~B}$ & $61 \mathrm{C}^{*}$ \\
\hline
\end{tabular}

Letras maiúsculas diferentes em uma mesma linha indicam diferença estatística entre diluidores para cada temperatura (G1 $\times$ G3 $\times$ G5) e (G2 x G4 x G6) $(p<0,05)$.

* em uma mesma linha indicam superioridade estatística entre temperaturas de descongelação para cada diluidor (G1xG2), (G3xG4) e (G5xG6) $(p<0,05)$ todos os grupos estudados a despeito do meio diluidor utilizado ou da temperatura em que se realizou a descongelação (Tabelas 1,2 , e 3).

Quando avaliamos amostras processadas em diferentes meios diluidores e descongeladas em cada temperatura distintamente, verificamos que não houve diferença estatística entre os grupos quanto à motilidade e que, quanto ao vigor espermático houve tendência estatística de superioridade dos grupos imediatamente após a descongelação a $72^{\circ} \mathrm{C}$ (M3) (Tabela 2). Comparando-se os diluidores em cada temperatura de descongelação $(\mathrm{G} 1 \mathrm{x} \mathrm{G} 3 \mathrm{x} \mathrm{G} 5$ e $\mathrm{G} 2 \times \mathrm{g} 4 \mathrm{xG6}$ ) observamos que houve tendência a superioridade quanto ao vigor espermático no grupo congelado sob o protocolo M9 frente aos outros protocolos quando se descongelava o sêmen a $37^{\circ} \mathrm{C}$, concordando com os resultados de Cunha e Lopes ${ }^{23}$.

Após o teste de longevidade também não houve diferença estatística do vigor espermático entre os grupos, porém, houve tendência $(0,05<\mathrm{p}<0,10)$ do GG e do M9 a apresentarem os maiores valores, sendo que dentre estes dois grupos, o G4 (M9 descongelado a $72^{\circ} \mathrm{C}$ ) foi o que apresentou maiores valores.

A formação de espécies reativas de oxigênio é um dos fatores que age negativamente sobre a célula espermática durante o processo de refrigeração e congelação do sêmen ${ }^{18,24}$. Cunha ${ }^{18}$ e Papa et al. ${ }^{25}$ sugeriram que a glicina exerce uma função de antioxidante quando presente no meio diluidor. Este aminoácido está presente no diluidores GG e M9.

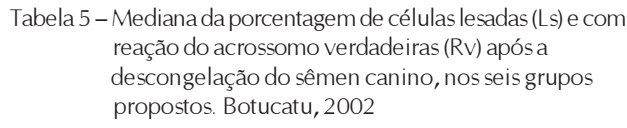

Tabela 5 - Mediana da porcentagem de células lesadas (Ls) e com reação do acrossomo verdadeiras (Rv) após a descongelação do sêmen canino, nos seis grupos propostos. Botucatu, 2002

\begin{tabular}{|c|c|c|c|c|c|c|}
\cline { 2 - 7 } \multicolumn{1}{c|}{} & G1 & G2 & G3 & G4 & G5 & G6 \\
\hline Rv & 8 & 8 & 0 & 1 & $10^{*}$ & $\underset{*}{22,5}$ \\
\hline $\mathrm{Ls}$ & 7,5 & 16 & 0 & 0,5 & 1 & 1 \\
\hline
\end{tabular}

* indica significa diferença estatística significativa 
O diluidor M9 além da glicina possui uma gama de outros aminoácidos em sua composição, entre eles a cisteina que, como descrito por Bilodeau et al. ${ }^{24}$, é um precursor intracelular da glutationa, que, por sua vez, é um antioxidante capaz de reagir e anular diretamente espécies reativas do oxigênio o que pode justificar uma tendência a superioridade deste grupo frente aos congelados sob outros protocolos e descongelado a $37^{\circ} \mathrm{C}$ (Tabela 2).

Verificamos, no entanto, superioridade estatística quanto à porcentagem de membranas íntegras nos grupos congelados com TRIS após o teste de longevidade (M4), tanto nos grupos descongelados a $37^{\circ} \mathrm{C}(\mathrm{G} 1=\mathrm{G} 3 \leq \mathrm{G} 5)$ como nos grupos descongelados a $72^{\circ} \mathrm{C}$ (G2 $\leq$ G4 $\leq$ G6) (Tabela 3). Entretanto, nos grupos congelados com meio TRIS, verificou-se, também, uma diminuição significativa da linearidade do movimento espermático após a descongelação, tanto no grupo descongelado com $37^{\circ} \mathrm{C}$ como no descongelado com $72^{\circ} \mathrm{C}$ (Tabela 4). Observamos ainda uma quantidade significativamente maior de células exibindo reação do acrossomo verdadeira nestes mesmos grupos após a descongelação (G5 e G6) (Tabela 5).

Rigau et al. ${ }^{26}$ avaliaram o efeito da glicose e o da frutose sobre o padrão de motilidade do sêmen canino. Estes autores concluíram que a frutose produz um movimento espermático mais rápido e linear enquanto a incubação em glicose induz a movimentos oscilatórios semelhantes aos verificados após a hiperativação espermática. O meio TRIS contém apenas glicose em sua constituição enquanto o GG possui frutose e glicose. O que pode justificar a diferença de padrão de motilidade entre estes dois meios.

O EDTA, que está presente no M9, é um quelante de cálcio, sendo assim, a concentração do cálcio em presença desta substância no meio é diminuída. A entrada de cálcio na célula espermática leva à capacitação espermática que, por conseguinte, induzirá movimentos oscilatórios assim como desencadeará uma série de alterações que culminará na reação do acrossomo ${ }^{19}$. O meio M9 provavelmente exerceu uma maior proteção às células espermáticas, diminuindo os risco do inicio do processo de capacitação.

Podemos aqui especular que o diluidor TRIS, nas condições utilizadas neste experimento, não foi tão eficiente quanto o GG e o M9 em proteger o metabolismo espermático e as membranas espermáticas, permitindo o inicio do processo de capacitação, uma vez que, algumas das características observadas com o desencadeamento deste processo são: o aumento do influxo de cálcio com alteração do padrão de movimento espermático (alteração linearidade do movimento) (Tabela 4) e ainda um aumento da fluidez da membrana acrossomal externa sem que tenha havido a ruptura da membrana plasmática externa (reação acrossomal verdadeira) (Tabela 5), explicando ainda, a maior quantidade de células espermáticas integras presentes nos grupo G5 e G6 quando comparado aos demais ${ }^{3,19}$ (Tabela 3).

O teste de verificação do estado acrossomal utilizando-se Fitc-PNA e iodeto de propídio sob microscopia de epifluorescência não tinha sido ainda descrita para a espécie canina, no que se refere ao conhecimento dos autores deste trabalho. $\mathrm{O}$ Fitc-PNA liga-se a radicais glicosilados que são expostos na membrana acrossomal externa com alteração de permeabilidade, que pode ser considerado um dos eventos precursores da reação do acrossomo ${ }^{19}$, permitindo assim, a avaliação qualitativa do estado do acrossomal de uma amostra de sêmen. Entretanto, o Fitc-PNA liga-se também a resíduos de açúcares presentes nos meios diluidores, e ainda, a maioria das lecitinas liga-se ao leite, gema de ovo e a outras partículas presentes no plasma seminal dificultando a avaliação ${ }^{13}$. Considerando-se este fato, a realização de centrifugação e lavagem das amostras de sêmen antes da realização desta avaliação, que não foi 
realizada neste experimento e que poderia evitar a coloração de resíduos de diluidores deveria ser ponderada. Devemos ainda ressaltar que a avaliação do estado acrossomal em nosso experimento foi realizada apenas em parte dos animais estudados (30 palhetas, provenientes de 5 dos 9 doadores).

Os grupos congelados em GG conservaram melhor MT, MP, VAP e LIN após a descongelação, estes achados concordam com os de Lopes e $\mathrm{Papa}^{27} \mathrm{e}$ com os de Barnabé e Barnabé ${ }^{28}$ e ainda com as conclusões de Rigau et al. ${ }^{26}$, já que este é o meio diluidor GG é o único que leva frutose em sua composição. Verificamos ainda que o grupo congelado com GG e descongelado a $72^{\circ} \mathrm{C}$ apresentou sempre a melhor qualidade de movimento espermático (Tabela 4).

Após a avaliação estatística dos dados do vigor espermático, observamos a tendência a superioridade $(0,05<\mathrm{p}<, 10)$ das amostras onde a descongelação foi realizada a $72^{\circ} \mathrm{C}$ por 8 segundos nos grupos congelados em GG e em TRIS (Tabela 2). Após o teste de longevidade não se verificou diferença entre os grupos quando avaliadas as temperaturas de descongelação em cada diluidor $(\mathrm{G} 1=\mathrm{G} 2)(\mathrm{G} 3=\mathrm{G} 4)(\mathrm{G} 5=\mathrm{G} 6)$.

Quanto à integridade das membranas avaliada entre as diferentes temperaturas de descongelação em um mesmo meio $(\mathrm{G} 1=\mathrm{G} 2)(\mathrm{G} 3=\mathrm{G} 4)(\mathrm{G} 5=\mathrm{G} 6)$ verificamos que não houve diferença estatística significativa entre as temperaturas ou entre os meios diluidores após a diluição das amostras, período de equilíbrio e descongelação (Tabela 3). Após o teste de longevidade quando comparamos as amostras congeladas em meio M9 (G3 e G4) verificamos que as descongeladas a $72^{\circ} \mathrm{C}$ eram superiores àquelas descongeladas a $37^{\circ} \mathrm{C}$ corroborando com os achados descritos por Cunha e Lopes ${ }^{23}$.

Foi observada grande diferença quanto aos valores verificados pela avaliação da motilidade subjetiva e a avaliação computadorizada das amostras de sêmen (Tabelas 1 e 4). Provavelmente tal fato ocorreu devido ao tempo decorrido entre a avaliação subjetiva da motilidade (primeiro item a ser avaliado imediatamente após as descongelações) e a avaliação computadorizada do movimento espermático (último item a ser observado, geralmente 30 min. após as descongelações). Um decréscimo de $\sim 45 \%$ na motilidade espermática após 60 min. de incubação do sêmen canino a $37^{\circ} \mathrm{C}$ foi também descrito por Rigau et al..$^{26}$.

Verificamos que a motilidade progressiva (MP) a velocidade média (VAP) e a linearidade do movimento (LIN) foram maiores com a descongelação a $72^{\circ} \mathrm{C}$, nos grupos onde os diluidores GG e TRIS foram utilizados (Tabela 4).

De uma maneira geral, o movimento espermático apresentou-se superior para o grupo congelado em meio Glicina Gema e descongelado a $72^{\circ} \mathrm{C}$ por 8 segundos (G2), porém, testes in vivo de fertilidade deverão ser realizados para que os resultados destas avaliações in vitro sejam comprovados.

A partir dos resultados obtidos neste experimento, concluímos que as células espermáticas caninas descongeladas a $72^{\circ} \mathrm{C}$ por 8 segundos apresentaram um maior somatório de bons resultados quanto aos testes de viabilidade espermática in vitro aqui empregados.

\section{Agradecimentos}

À FAPESP - Fundação de Amparo à Pesquisa do Estado de São Paulo pelo apoio financeiro concedido para a realização deste trabalho.

À Professora Fernanda Landim Alvarenga (UNESP - Botucatu) pela orientação quanto a padronização do teste de avaliação do estado acrossomal para a espécie canina. 


\title{
Effect of three different diluent on canine semen submitted to two thawing protocols
}

\begin{abstract}
To verify the effect of three different extenders and two thawing temperatures on frozen-thawed canine sperm characteristics, one ejaculate from nine dogs were separately collected and processed $(n=9)$. Each ejaculate was divided into 3 equal samples and centrifuged. The pellets were re-suspended using: $1^{\text {st }}$ pellet-Glicyne-egg yolk extender (GEY), $2^{\text {nd }}$ pellet - TRIS extender and $3^{\text {rd }}$ pellet - M9 extender and all samples were filled in $0.5 \mathrm{ml}$ straws. A total of 84 straws $(28$ for each protocol) were done. The sperm motility, vigour and plasma membrane integrity from each protocol were immediately evaluated (M1) and the straws were brought to a refrigerator at $5^{\circ} \mathrm{C}$ for 60 minutes. After that, a sample from each protocol was warmed up in a water bath $37^{\circ} \mathrm{C}$ for $5 \mathrm{~min}$. and sperm motility, vigour and plasma membrane integrity were evaluated (M2). The straws were frozen in liquid nitrogen. One straw from each protocol was thawed at $37^{\circ} \mathrm{C}$ for $30 \mathrm{sec}$ and another at $72^{\circ} \mathrm{C}$ for $8 \mathrm{sec}$ and the sperm motility; vigour, CASA and plasmatic membrane integrity and acrossomal status using FITC-PNA stain were evaluated (M3). Plasma membrane, sperm motility and vigour were evaluated after a 1-hour incubation at $37^{\circ} \mathrm{C}$ (M4). Statistical analysis showed that the higher temperature had positive effect on froze-thawed canine sperm characteristics. The best results were taken when canine semen was frozen in GEY extender and thawed $72^{\circ} \mathrm{C}$ for $8 \mathrm{sec}$.
\end{abstract}

\section{Referências}

1 PEÑA, A. I. Flow cytometry in the assessment of fresh and frozen-thawed dog semen, and the effects of different cryopreservation methods on post-thaw sperm survival and longevity. 2000. $46 \mathrm{f}$. Thesis (Doctoral) - Swedish University of Agricultural Sciences, Uppsala, 2000.

2 WATSON, P. F. Recents developments and concepts in the cryopreservation of spermatozoa and the assesment of their post-thawing function. Reprod. Fertil. Dev., v. 7, p. 871-891, 1995.

$3 \mathrm{HOLT}, \mathrm{W}$. V. Basic aspects of frozen storage of semen. Anim. Reprod. Sci., v. 62, p. 3-22, 2000.

4 ROTA, A. Studies on preservation capacitation and fertility of dog spermatozoa. 1998. $43 \mathrm{f}$. Dissertation (Doctor's) - Swedish University of Agricultural Sciences, Uppsala, 1998.

5 BATEMAN, H. L. Effects of semen extender composition and cooling methods on canine sperm function and cryo-survival. 2001. $92 \mathrm{f}$. Thesis (Master of Science) - University of Guelph, 2001. Guelph.

6 POMMER, A. C.; RUTLLANT, J.; MEYERS, S. A. The role of osmotic resistance on equine spermatozoal function. Theriogenology, v. 58, p. 1373-1384, 2002.

7 ENGLAND, G. C. W. The criopreservation of dog semen. 1992. $152 \mathrm{f}$. Thesis submitted for Felloship of Royal College of Veterinary Surgeons, London, 1992.

8 LINDE-FORSBERG, C. Artificial insemination with fresh, chilled extended and frozen-thawed semen in dog. Semin. Vet. Surg. Small Anim., v. 10, p. 58, 1995.

9 OLAR, T. T. Cryopreservation of dog semen. 1984. 158 f. PhD Thesis, Colorado State University, Fort Collins, 1984.

10 IVANOVA-KICHEVA, M. G. et al. Effect of thawing regimens on the morphofunctional state of canine spermatozoa. Theriogenology, v. 44, p. 563-569, 1995.

11 STRÖM HOLST, B. In vitro characterisation of cryopreserved canine spermatozoa with special reference to post-thaw time and zona pellucida binding capacity. 1999. 40 f. Dissertation (Doctor's), Swedish University of Agricultural Sciences, Uppsala, 1999.

12 COLÉGIO BRASILEIRO DE REPRODUÇÃO ANIMAL. Manual para exame andrológico e avaliação de sêmen animal. Belo Horizonte, 1996. 65 p. Elaborado conforme convênio CBRA/MA n. 017/1996.

13 KIRK, E. S. Flow cytometric evaluation of stallion sperm. 2001. $131 \mathrm{f}$. Thesis (Master of Science) - Colorado State University, Fort Collins, 2001. 
14 IGUER-OUADA, M.; VERSTEGEN, J. Validation of sperm quality analyzer (SQA) for dog semen analysis. Theriogenology. v. 55, p. 1143-1158, 2001.

$15 \mathrm{BLACH}, \mathrm{E}$. L. et al. Changes in quality of stallion spermatozoa during cryopreservation: plasma membrane integrity and motion characteristics. Theriogenology, v. 31, p. 283-299, 1989.

16 HARRISON, R. A. P.; VICKERS, S. E. Use of fluorescent probes to asses membrane integrity in mammalian spermatozoa. J. Reprod. Fertil., v. 88, p. 343-352, 1990

17 KUMI-DIAKA, J. Subjecting canine semen to the hypo-osmotic test. Theriogenology, v. 39, p. 1279-1289, 1993.

18 CUNHA, I. C. N. Estudo da viabilidade do processo de refrigeração do sêmen canino utilizando-se diluidores à base de leite e glicina-gema. 1997. $124 \mathrm{f}$. Dissertação (Mestrado em Reprodução Animal) Faculdade de Medicina Veterinária e Zootecnia, Universidade Estadual Paulista, Botucatu, 1997.

19 CROSS, N. Role of cholesterol in sperm capacitation. Biol. Reprod., v. 59, p. 7-11, 1998

20 CROSS, N. L.; WATSON, K. Assessing acrossomal status of bovine sperm using fluoresceinated lectins. Theriogenology, v. 42, p. 89-98, 1994.

21 MEDEIROS, A. S. L. et al. Avaliação da integridade acrossomal de espermatozóides de garanhões criopreservados com crioprotetores a base de amidas e glicerol. Rev. Bras. Reprod. Anim., v. 27, n. 3, p. 353 354, 2003.

22 KENNEY, R. M. et al. Minimal contamination techniques for breeding mares: technique and preliminary findings. In: ANNUAL CONVENTION AMERICAN ASSOCIATION EQUINE PRACTITIONERS, 21., 1975. Boston. Proceedings... Boston, 1975. p. 327.

23 CUNHA, I. C. N.; LOPES, M. D. Efeito de diferentes períodos de equilíbrio e meios diluidores sobre o sêmen canino congelado e descongelado. Rev. Bras. de Reprod. Anim., v. 25, n. 3, p. 470-471, 2001.

24 BILODEAU, J. F. et al. Thiols prevent $\mathrm{H}_{2} \mathrm{O}_{2}$-mediated loss of sperm motility in cryopreserved bull semen. Theriogenology, v. 56, p. 275-286, 2001.

25 PAPA, F. O. et al. Glicina-Gema: Proposta de um novo diluidor para congelação de sêmen eqüino. In: SIMPÓSIO INTERNACIONAL DE REPRODUÇÃO ANIMAL, 5., 1993. Portugal. Anais... Portugal, 1993. v. 2 , p. $378-384$.

26 RIGAU, T. et al. Effects of glucose and fructose on motility patterns of dog spermatozoa from fresh ejaculates. Theriogenology, v. 56, p. 801-815, 2001.

27 LOPES, M. D.; PAPA, F. O. Effects of diferent diluents and method of centrifugation for canine semen congelation. In: CONGRESS OF THE WORDL SMALL
ANIMAL VETERINARY ASSOCIATION, 23., 1998. Buenos Aires. Proceedings... Buenos Aires, 1998. p. 799.

28 BARNABÉ, V. H.; BARNABÉ, R. C. Effect of caffeine citrate and heparin on post-thawed motility and on acrossomal cap of buffalo: assay for in vitro fertilization technique. Hum. Sperm Acros. Reac., v. 236, p. 430, 1995. 